\title{
POURQUOI CUBA EST-ELLE DEVENUE UN PROBLÈME DIFFICILE POUR LA GAUCHE ?
}

Boaventura de Sousa Santos

\author{
La Découverte | «Mouvements »
}

2009/4 n $60 \mid$ pages 99 à 112

ISSN 1291-6412

ISBN 9782707158932

Article disponible en ligne à l'adresse :

http://www.cairn.info/revue-mouvements-2009-4-page-99.htm

\section{Pour citer cet article :}

Boaventura de Sousa Santos, « Pourquoi Cuba est-elle devenue un problème difficile pour la gauche ? », Mouvements 2009/4 (n 60), p. 99-112.

DOI 10.3917/mouv.060.0099

Distribution électronique Cairn.info pour La Découverte.

(C) La Découverte. Tous droits réservés pour tous pays.

La reproduction ou représentation de cet article, notamment par photocopie, n'est autorisée que dans les limites des conditions générales d'utilisation du site ou, le cas échéant, des conditions générales de la licence souscrite par votre établissement. Toute autre reproduction ou représentation, en tout ou partie, sous quelque forme et de quelque manière que ce soit, est interdite sauf accord préalable et écrit de l'éditeur, en dehors des cas prévus par la législation en vigueur en France. Il est précisé que son stockage dans une base de données est également interdit. 
THÈMES 


\section{Pourquoi Cuba est-elle devenue un problème difficile pour la gauche?}

\author{
PAR \\ Boaventura de \\ Sousa Santos *
}

\author{
Si la singularité de la " voie cubaine au socialisme " représente \\ un problème pour la gauche, la rénovation de cette dernière \\ passe selon Boaventura de Sousa Santos par un nouveau type de \\ partage d'expériences avec Cuba et par une transition ouverte à \\ l'expérimentation.
}

* Né à Coimbra (Portugal) en 1940, est l'un des grands intellectuels de référence dans les discussions à propos du changement social et politique en Amérique Latine. Il participe depuis le début à l'expérience du Forum Social Mondial et dirige le Centro de estudos sociais de l'Université de Coimbra.

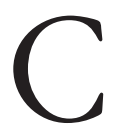
ette question peut paraître étrange et beaucoup vont penser que la formulation inverse aurait peut-être plus de sens : pourquoi la gauche est-elle devenue un problème difficile pour Cuba? En fait, la révolution cubaine occupe, dans la pensée et la pratique de la gauche au cours du $\mathrm{XX}^{\mathrm{e}}$ siècle, une place incontournable. Elle est d'autant moins contournable si l'on ne se concentre pas tant sur la société cubaine en elle-même, que sur la contribution de Cuba aux relations entre les peuples, en particulier à travers les nombreuses démonstrations de solidarité internationaliste dont a témoigné la Révolution cubaine ces 50 dernières années. L'Europe et l'Amérique du nord seraient peut-être devenues ce qu'elles sont sans la Révolution cubaine, mais on ne peut pas en dire autant de l'Amérique latine, de l'Afrique ou de l'Asie, régions de la planète où vivent près de $85 \%$ de la population mondiale. La solidarité internationaliste dont Cuba a été le protagoniste s'est étendue en cinq décennies dans les domaines les plus divers, politique, militaire, social et humanitaire.

\section{- Qu'est-ce que "la gauche ",}

\section{qu'est-ce qu' " un problème difficile " ?}

Je pense malgré tout que la question à laquelle j'essaie de répondre dans ce texte a un sens. Mais avant de tenter une réponse, il faut faire quelques précisions. Premièrement, la question pourrait suggérer que seule Cuba a évolué et est devenue problématique ces 50 dernières années et que, au contraire, la gauche qui l'interpelle aujourd'hui est la même qu'il y a 50 ans. Rien ne serait plus faux. Cuba, comme la gauche, a beaucoup évolué lors de ce demi-siècle et ce sont leurs voies divergen- 
tes de leurs évolutions respectives qui ont créé le "problème difficile ". Il est vrai que Cuba a essayé activement de changer la scène internationale de façon à rendre plus justes les relations entre les peuples. Mais il est tout aussi vrai que les conditions externes hostiles dans lesquelles la Révolution cubaine a été obligée d'évoluer ont empêché que le potentiel de rénovation de la gauche dont la Révolution était porteuse en 1959 se réalise pleinement. Ainsi, la gauche mondiale ne s'est pas rénovée, ces 50 dernières années, à partir du legs de la Révolution cubaine, mais à partir d'autres référents. La solidarité internationale cubaine a pu maintenir une vitalité de loin supérieure à la formule interne à Cuba.

Deuxièmement, je dois préciser ce que j'entends par "gauche " et par "problème difficile". La gauche signifie l'ensemble des théories et des pratiques transformatrices qui, ces 150 dernières années, ont résisté à l'expansion du capitalisme et au type de relations économiques, sociales, politiques et culturelles qu'il engendre. Elles procèdent de la croyance en la possibilité d'un futur postcapitaliste, d'une société alternative, plus juste parce qu'orientée vers la satisfaction des besoins réels des peuples, et plus libre parce que centrée sur la réalisation des conditions de l'exercice effectif de la liberté. On a donné à cette société alternative le nom générique de socialisme. Je soutiens que pour cette gauche, dont la théorie et la pratique ont beaucoup évolué ces 50 dernières années, Cuba constitue un "problème difficile". Pour la gauche qui a éliminé de son horizon le socialisme ou le postcapitalisme, Cuba n'est même pas un problème, mais une cause perdue. Ce n'est pas de cette gauche dont je m'occupe ici.

Par " problème difficile " j'entends un problème qui nous place dans une alternative entre deux positions diamétralement opposées face à l'objet qu'elle interroge, en l'occurrence Cuba. Les deux positions écartées par l'idée du " problème difficile "sont : Cuba est une solution sans problème, Cuba est un problème sans solution. Qualifier Cuba de "problème difficile " pour la gauche signifie accepter trois idées : 1) dans les conditions internes actuelles, Cuba a cessé d'être une solution viable de gauche ; 2) les problèmes qu'elle affronte, sans être insurmontables, sont difficiles à résoudre ; 3) si de tels problèmes étaient résolus en termes d'un horizon socialiste, Cuba pourra redevenir un moteur de la rénovation de la gauche. Ce sera alors un Cuba différent, qui engendrera un socialisme différent de celui qui a échoué au $\mathrm{XX}^{\mathrm{e}}$ siècle, et contribuera ainsi à la rénovation urgente de la gauche. Sans cette rénovation, la gauche n'entrera jamais au $\mathrm{XXI}^{\mathrm{e}}$ siècle.

\section{- La résistance et l'alternative}

Une fois faites ces précisions, le "problème difficile " peut se formuler ainsi : tous les processus révolutionnaires modernes sont des processus de rupture qui se fondent sur deux piliers : la résistance et l'alternative. L'équilibre entre les deux est fondamental pour éliminer l'ancien autant qu'il est nécessaire, et faire fleurir le nouveau autant que possible. En raison des conditions externes hostiles dans lesquelles le processus révolutionnaire cubain a évolué - l'embargo illégal des États-Unis, la solution 


\section{Dans tout processus révolutionnaire, le premier acte des révolutionnaires après le succès de la révolution consiste à éviter qu'il n'y ait d'autres révolutions.}

soviétique forcée dans les années 1970, l'ajustement drastique provoqué par la disparition de l'Union soviétique dans les années 1990 cet équilibre n'a pas été atteint. La résistance a fini par s'imposer face à l'alternative. Ce faisant, l'alternative n'a pas pu s'exprimer selon sa propre logique (affirmation du nouveau) et a dû, au contraire, se

soumettre à la logique de la résistance (la négation de l'ancien). Le résultat de cette situation est que l'alternative est restée l'otage d'une norme qui lui était étrangère. C'est-à-dire qu'elle ne s'est jamais transformée en véritable solution neuve, consolidée, créatrice d'une nouvelle hégémonie et, pour autant, capable de développement endogène selon une logique interne de rénovation (nouvelles alternatives au sein de l'alternative). Par conséquent, les ruptures avec les passés successifs de la Révolution ont toujours été moins endogènes que la rupture avec le passé prérévolutionnaire. Le caractère endogène de cette dernière a pu justifier l'absence de ruptures endogènes avec les passés plus récents, y compris quand ceux-ci étaient notoirement problématiques.

En raison de ce relatif déséquilibre entre résistance et alternative, l'alternative a toujours été proche de la stagnation, et cette stagnation a toujours été déguisée par la vitalité continue et noble de la résistance. Cette prédominance de la résistance a fini par lui attribuer un " excès diagnostique ": la nécessité de la résistance pouvait être invoquée pour diagnostiquer l'impossibilité de l'alternative. Même si elle était factuellement erronée, une telle invocation a toujours été crédible.

\section{- Le charisme révolutionnaire et le système réformiste}

Le deuxième vecteur du "problème difficile " concerne la manière spécifiquement cubaine dont s'est manifestée la tension entre révolution et réforme. Dans tout processus révolutionnaire, le premier acte des révolutionnaires après le succès de la révolution consiste à éviter qu'il n'y ait d'autres révolutions. Avec cet acte commence le réformisme au sein de la révolution. Là réside la grande complicité - aussi invisible que décisive entre révolution et réformisme. Dans le meilleur des cas, cette complémentarité peut être atteinte par une dualité - toujours plus apparente que réelle - entre le charisme du leader, qui assure la continuation de la révolution, et le système politique révolutionnaire, qui assure la reproduction du réformisme. Le leader charismatique voit le système comme un carcan qui limite son impulsion révolutionnaire, et qui le pousse vers le changement ; tandis que le système voit le leader comme un ferment de chaos qui rend provisoires toutes les vérités bureaucratiques. Cette dualité créative a été pendant quelques années l'une des caractéristiques de la Révolution cubaine.

Cependant, avec le temps, la complémentarité vertueuse tend à se transformer en blocage réciproque. Pour le leader charismatique, le système, 
qui commence par être une limitation qui lui est extérieure, se convertit avec le temps dans sa deuxième nature. Il devient difficile de distinguer les limitations que créé le système de celles liées au leader même. Le système, à son tour, découvrant le succès du réformisme, finira par miner le charisme du leader, et s'autolimite donc pour empêcher que cela ne se produise. La complémentarité se transforme en un jeu d'autolimitations réciproques. Le risque étant que, loin de déboucher sur des développements complémentaires, elles produisent des stagnations parallèles.

Le rapport entre charisme et système tend donc à être très instable, tout particulièrement dans les phases de transition. Aurelio Alonso fait une distinction entre deux processus de transition en cours : l'un se réfère à la direction de la dynamique de changement au sein d'une "grande transition qui a commencé il y a un demi-siècle " et l'autre "se réfère au poids de la subjectivité : la question de l'empreinte que Fidel laissera dans l'imaginaire des Cubains des générations qui lui survivront ${ }^{1}$ ".

Le charisme, en lui-même, n'admet pas de transition. Aucun leader charismatique n'a de successeur charismatique. La transition ne peut avoir lieu que dans la mesure où le système remplace le charisme. Il faut pour cela que le système soit suffisamment réformiste pour affronter des sources de chaos très différentes de celles qui proviennent du leader. La situation se présente comme un dilemme lorsque la force du leader charismatique bloque objectivement le potentiel réformiste du système.

Ce vecteur du "problème difficile" peut se résumer ainsi : le futur socialiste de Cuba dépend de la force réformiste du système révolutionnaire. Néanmoins, cette force est une inconnue pour un système qui a toujours fait dépendre sa force du leader charismatique. Ce vecteur de la difficulté du problème explique le discours de Fidel à l'Université de La Havane du 17 novembre 2005. Dans la formule lapidaire de Fidel : " Ce pays-ci peut s'autodétruire, cette Révolution peut se détruire. Ceux qui ne peuvent pas la détruire, ce sont eux ; nous, en revanche, nous pouvons le faire, et ce serait notre faute. " Commentant cette intervention de Fidel, Aurelio Alonso écrit : "Qu'y a-t-il d'étrange au fait que la principale préoccupation de Fidel tourne autour du caractère réversible de notre propre processus ?" Et il répond de façon catégorique : "Fidel estime que si la révolution ne peut être détruite de l'extérieur, elle peut se saborder ellemême. Il signale la corruption comme le mal pouvant provoquer cette destruction. Je pense qu'il a raison, mais qu'il n'a pas tout dit. Je me demande en plus si l'écroulement du système soviétique était, essentiellement, un effet de la corruption, même si la corruption faisait certainement partie de la structure des déviations. À mon avis le socialisme peut être renversé par la bureaucratie et l'absence de démocratie aussi bien que par la corruption. Je ne parle pas là de systèmes électoralistes, des confrontations pluripartidaires, des luttes de campagne, ni des alternances au pouvoir. Je parle de la démocratie, que nous n'avons pas été capables de créer sur Terre, bien que nous croyions tous savoir ce que c'est ${ }^{2}$ ".

Les deux versants du "problème difficile " - déséquilibre entre résistance et alternative et entre charisme et système - sont intimement liés. La pré-
1. «Continuidad y transición : Cuba en el 2007", Le Monde Diplomatique, édition colombienne, Bogotá, avril 2007.

2. Cf. "Una mirada rápida al debate sobre el futuro de Cuba " $\mathrm{La}$ Jiribilla, 17 mai 2006. 
3. Cf. Nature, Society and Thought, vol. 19, 2006, p. 20.
4. Dans le cas du marxisme il y a eu beaucoup de créativité pour adapter la théorie à des réalités non européennes qui n'avaient pas été systématiquement analysées par Marx. Mais, pendant longtemps les orthodoxies politiques n'ont pas permis de transformer cette créativité en action politique. Les auteurs les plus créatifs ont été persécutés.

dominance de la résistance sur l'alternative a été simultanément le produit et le producteur de la prédominance du charisme sur le système.

\section{Q Que faire ?}

La discussion précédente démontre que Cuba est un "problème difficile " pour cette gauche qui, sans abandonner l'horizon du postcapitalisme ou du socialisme, a beaucoup évolué ces 50 dernières années. Des lignes principales de cette évolution le peuple cubain pourrait tirer des propositions pour trouver la solution du problème, en dépit de sa complexité. Autrement dit, la Révolution cubaine, qui a tant contribué à la rénovation de la gauche, surtout lors de sa première décennie, pourrait maintenant bénéficier aussi de la rénovation de la gauche qui s'est produite depuis lors. Ce faisant, elle assumera de nouveau, dialectiquement, un rôle actif dans la rénovation de la gauche. Résoudre le " problème difficile " impliquerait donc concrétiser avec succès le mouvement dialectique suivant : rénover Cuba en rénovant la gauche ; rénover la gauche en rénovant Cuba.

\section{- Les principaux chemins de la rénovation de la gauche socialiste lors de ces $\mathbf{5 0}$ dernières années}

1. Lors des 50 dernières années l'écart entre théorie de gauche et pratique de gauche s'est élargi, avec des conséquences très spécifiques pour le marxisme. Alors que la théorie de la gauche critique s'est développée, principalement, à partir de la deuxième moitié du XIX ${ }^{\mathrm{e}}$ siècle, dans cinq pays du Nord de la planète (Allemagne, Angleterre, Italie, France et ÉtatsUnis), et en tenant compte en particulier des réalités des sociétés des pays capitalistes développés, les pratiques de gauche les plus créatives se sont manifestées dans le Sud, et ont eu pour protagonistes des classes ou groupes sociaux " invisibles ", ou en tout cas semi-visibles pour la théorie critique et même pour le marxisme, tels que les peuples colonisés, les peuples indigènes, les paysans, les femmes, les afro-descendants, etc. La créativité théorique initiale de la Révolution cubaine réside véritablement dans ce fait. Les conditions extérieures drastiques auxquelles la révolution était sujette ont fini par confisquer une partie de cette créativité. Pour cette raison, Cuba a été obligée de recourir à une conception du marxisme qui dépendait de la réalité du bloc soviétique, qui ressemblait peu à la réalité cubaine. Lors de la IIIe Conférence Internationale sur " L'œuvre de Karl Marx et les défis du XXI e siècle ", tenue à La Havane le 3 mai 2006, Ricardo Alarcón de Quesada a affirmé : "La transformation de l'expérience soviétique en paradigme pour ceux qui, en d'autres lieux, livraient leurs propres luttes anticapitalistes, et l'obligation impérieuse de défendre [l'URSS] contre des ennemis puissants et acharnés, a amené à la subordination d'une grande partie du mouvement révolutionnaire à la politique et aux intérêts de l'URSS ${ }^{3}$ ". Une disjonction entre théorie et pratique s'est ainsi créée. Cette disjonction domine notre condition théorico-politique d'aujourd'hui, à savoir : une théorie semi-aveugle avance parallèlement à une pratique semi-invisible ${ }^{4}$. Or, une théorie semi-aveugle ne sait pas 
commander et une pratique semi-invisible ne sait pas se mettre en valeur. En perdant dans la pratique son rôle d'avant-garde - puisque beaucoup de ce qui se passait lui échappait complètement ${ }^{5}$ - elle a graduellement abandonné le statut de théorie d'avant-garde tout en revêtant un statut complètement nouveau et inconcevable dans la tradition nordo-centrique de la gauche : le statut d'une théorie d'arrière-garde.

Dans le sens que je lui attribue, la théorie de l'arrière-garde signifie deux choses. D'une part, c'est une théorie qui ne guide pas sur la base de principes ou de lois générales qui régiraient théoriquement la totalité historique, mais sur la base d'une analyse constante, critique et ouverte, des pratiques de transformation sociale. Ainsi la théorie de l'arrière-garde se laisse surprendre par des pratiques de transformation progressistes, les accompagne, les analyse, et tente de s'enrichir grâce à elles, et cherche en elles les critères pour approfondir et élargir les luttes sociales les plus progressistes. D'autre part, une théorie de l'arrière-garde regarde, dans ces pratiques transformatrices, autant les processus et acteurs collectifs les plus avancés que les plus retardataires, les plus timides voire au bord du renoncement. Comme le dirait le sous-commandant Marcos, c'est une théorie qui chemine avec ceux qui vont plus lentement, avec ceux de l'avant comme ceux de l'arrière, une théorie qui conçoit des avancées et des reculs, dans le cadre d'un processus dialectique nouveau. Ce dernier ne présuppose pas l'idée de totalité mais postule plutôt l'idée de différents processus de totalisation, toujours inachevés et toujours en concurrence. Suivant la leçon de Gramsci, ceci est le chemin pour créer une contre-hégémonie socialiste ou, comme dans le cas cubain, pour maintenir et renforcer une hégémonie socialiste.

Pour prendre un seul exemple, les peuples indigènes de l'Amérique latine, grands sujets invisibles ou oubliés de la théorie critique moderne (tout au plus sont-ils visibles en tant que paysans), ont été parmi les grands protagonistes des luttes progressistes de ces dernières décennies sur le continent. Dans la perspective de la théorie conventionnelle de l'avant-garde, toute cette innovation politique et sociale ne présenterait qu'un intérêt marginal, voire nul. Ainsi l'occasion est perdue d'apprendre des luttes des peuples indigènes, leurs notions d'économie et de bien-être (le suma kawsay des Quechuas ou le suma qamaña des Aymaras, c'est-àdire le "bien vivre") - notions aujourd'hui inscrites dans les constitutions de l'Équateur et de la Bolivie avec leurs conceptions des formes multiples de gouvernement et de démocratie (démocratie représentative et communautaire, comme cela est établi dans la nouvelle constitution de la Bolivie). L'incapacité à apprendre des nouveaux acteurs du changement finit par priver la théorie elle-même de toute pertinence.

2. La fin de la théorie de l'avant-garde marque la fin de toute forme d'organisation politique fondée sur elle, en particulier le parti d'avant-garde. Aujourd'hui les partis modelés par l'idée de la théorie de l'avant-garde ne sont ni des partis d'avant-garde ni d'arrière-garde (comme je la définis plus haut) : ce sont en fait des partis bureaucratiques qui, dans l'opposition, résistent fortement au statu quo, n'ayant pas d'autre choix ; et une
5. Autrement dit, la suprématie de l'intelligence et de l'audace politique sur la discipline, qui a été la marque de l'avantgarde, a fini par se transformer en son contraire : la suprématie de la discipline sur l'intelligence et l'audace comme moyen d'occulter ou contrôler la nouveauté des processus de transformation sociale non prévus par la théorie. 


\section{Démocratiser signifie transformer les rapports inégaux de pouvoir en rapports d'autorité partagée.}

fois au pouvoir, résistent fortement aux propositions d'alternatives. En remplacement du parti d'avantgarde il faudrait créer un parti (ou plus) d'arrière-garde pour accompagner la fermentation de l'activisme social générée lorsque les résultats de la participation démocratique populaire deviennent transparents, même pour ceux qui ne participent pas encore et qui sont ainsi attirés par la participation.

3. L'autre grande innovation des 50 dernières années a été la manière dont la gauche et le mouvement populaire se sont approprié les conceptions hégémoniques (libérales, capitalistes) de la démocratie et les ont transformées en conceptions contre-hégémoniques, participatives, délibératives, communautaires, radicales. Nous pouvons résumer cette innovation en affirmant que la gauche a enfin décidé de prendre la démocratie au sérieux (ce que la bourgeoisie n'a jamais fait, comme Marx l'avait bien noté). Prendre la démocratie au sérieux ne signifie pas seulement l'amener bien au-delà des frontières de la démocratie libérale mais aussi créer un concept de démocratie d'un type nouveau : la démocratie comme l'ensemble du processus de transformation des rapports de pouvoir inégaux en rapports d'autorité partagée.

La démocratie libérale, même quand elle n'est pas associée à la fraude, au rôle décisif de l'argent dans les campagnes électorales ou à la manipulation de l'opinion publique à travers le contrôle des moyens de communication, est une démocratie de basse intensité, puisqu'elle se limite à créer un îlot de relations démocratiques dans un archipel de despotismes (économiques, sociaux, raciaux, sexuels, religieux) qui contrôlent effectivement la vie des citoyens et des communautés. La démocratie doit exister bien au-delà du système politique, dans le système économique, dans les relations familiales, raciales, sexuelles, régionales, religieuses, et dans les relations de voisinage et de communauté. Le socialisme est la démocratie sans fin. Ainsi, l'égalité a plusieurs dimensions, et ne peut se réaliser pleinement qu'à la condition que nous luttions également pour la reconnaissance des différences - c'est-à-dire que nous transformions les différences inégales (qui créent des hiérarchies sociales) en différences égales (qui promeuvent la diversité sociale comme moyen pour éliminer les hiérarchies).

4. Dans les sociétés capitalistes les systèmes de rapports inégaux de pouvoir (d'oppression, de domination, d'exploitation, racisme, sexisme, homophobie, xénophobie, machisme) sont nombreux. Démocratiser signifie transformer les rapports inégaux de pouvoir en rapports d'autorité partagée. Puisque les rapports inégaux de pouvoir fonctionnent toujours en réseaux, il est rare qu'un citoyen, une classe ou un groupe ne soient victimes que d'un seul type de rapports inégaux séparément. De même, la lutte contre ces rapports inégaux doit s'organiser en réseaux, c'est-à-dire sur la base de larges alliances où il est impossible d'identifier un sujet historique privilégié, homogène, défini a priori en termes de 
classe sociale. D'où la nécessité du pluralisme politique et organisationnel dans le cadre constitutionnel sanctionné démocratiquement par le peuple souverain. Dans la société cubaine les rapports inégaux de pouvoir sont différents de ceux qui existent dans les sociétés capitalistes. Ils existent, bien qu'ils soient moins intenses, sont tout aussi multiples et fonctionnent eux aussi en réseau. La lutte contre ces rapports inégaux, une fois faites les adaptations nécessaires, doit également être guidée par le pluralisme social, politique et organisationnel.

5. Les nouvelles conceptions de la démocratie et de la diversité sociale, culturelle et politique, en tant que piliers de la construction d'un socialisme viable et en mesure de se reproduire (auto-sustentado), exigent que l'on repense radicalement la centralité monolithique de l'État, ainsi que l'homogénéité supposée de la société civile'.

\section{- Pistes pour une discussion en vue de contribuer à un futur socialiste viable à Cuba}

1. Cuba est peut-être le seul pays au monde où les conditions externes ne sont pas une excuse pour l'incompétence ou la corruption des leaders. Elles constituent un fait cruel et décisif mais cela ne signifie pas qu'il n'existe aucune marge de manœuvre. Elle pourrait même s'accrôtre avec la crise du néolibéralisme et les changements stratégiques prévisibles à court terme. Cette opportunité ne doit pas être gaspillée par le refus d'examiner les alternatives, même si celles-ci sont occultées par faux héroïsme ou une attitude de résistance. Nous ne pouvons désormais plus courir le risque de laisser la résistance dominer l'alternative. Si cela se produisait, il n'y aurait plus de résistance.

2. Le régime cubain a porté à sa limite la tension possible entre légitimation idéologique et conditions matérielles de vie. Désormais les changements qui comptent seront ceux qui transformeront les conditions matérielles de vie de l'immense majorité de la population. Dès lors, la démocratie de ratification, si elle doit continuer d'exister, ne ratifiera plus l'idéologie que dans la mesure où la ratification aura des traductions concrètes. Dans le cas contraire, la ratification ne signifierait plus le consentement mais la résignation.

3. La temporalité à long terme du changement civilisationnel sera pour un temps subordonnée à la temporalité immédiate des solutions urgentes.

4. Une société capitaliste n'est pas capitaliste parce que tous les rapports économiques et sociaux le sont, mais parce que les rapports capitalistes déterminent le fonctionnement de tous les autres rapports économiques et sociaux qui existent dans la société. Inversement, une société socialiste n'est pas socialiste parce que tous les rapports sociaux et économiques sont socialistes, mais parce que ceux-ci déterminent le fonctionnement de tous les autres. En ce moment à Cuba il y a une situation sui generis. D'une part, un socialisme formellement monolithique n'encourage pas l'émergence de rapports non capitalistes d'un type nouveau et ne peut pas déterminer de façon créative les rapports capitalistes, bien que parfois il vive aisément avec ceux-ci, jusque dans la corruption dénoncée de façon
6. Pour une vision lucide de la société civile à Cuba, voir "Sociedad civil en Cuba: iun problema de geometría? Entrevista con el sociólogo cubano Aurelio Alonso", dans la revue Enfoques $\mathrm{n}^{\circ}$ 23, La Havane, décembre 2008 
opportune par Fidel. Et, d'autre part une forme de capitalisme sauvage et clandestine, ou semi-clandestine, s'avère difficile à contrôler. Le terrain n'est ainsi pas propice au développement d'autres rapports économiques et sociaux de type coopératif ou communautaire, dont on pourrait attendre beaucoup. De ce point de vue le peuple cubain devrait étudier et évaluer très attentivement les systèmes économiques inscrits dans la constitution du Venezuela et dans celles d'Équateur de la Bolivie, récemment approuvées, ainsi que les expériences de transformation respectives de ces pays. Il ne s'agit pas de copier des solutions mais d'apprécier les chemins de la créativité de la gauche latino-américaine pendant ces dernières décennies. L'importance de cet apprentissage est implicite dans la reconnaissance des erreurs passées, exprimée avec force par Fidel dans le discours à l'Université de La Havane déjà mentionné : "J'ai tiré une conclusion au bout de bien des années : parmi les nombreuses erreurs que nous avons tous commises, la plus grosse a été de croire que quelqu'un savait ce qu'était le socialisme, ou comment on construit le socialisme".

5. Du point de vue du citoyen, la différence entre un socialisme inefficace et un capitalisme injuste est sans doute plus petite qu'on ne croit. Une relation de domination (basée sur un pouvoir politique inégal) peut avoir dans la vie quotidienne des personnes des conséquences étrangement semblables à celles d'une relation d'exploitation (basée sur l'extraction de la plus-value).

\section{- Un vaste et passionnant champ d'expérimentation sociale et politique à partir duquel Cuba peut contribuer à la rénovation de la gauche mondiale}

1. Démocratiser la démocratie. Contre les théoriciens libéraux, qui soutiennent que la démocratie est la condition de tout le reste, j'ai soutenu qu'il existe des conditions pour que la démocratie soit pratiquée réellement (genuinamente). J'ose dire que Cuba pourrait être l'exception de ma règle : je crois qu'à Cuba la démocratie radicale, contre-hégémonique, non libérale, est la condition de tout le reste. Pourquoi ? La crise de la démocratie libérale est aujourd'hui plus évidente que jamais. Il est chaque jour plus évident que la démocratie libérale ne garantit pas les conditions de survie face aux multiples " fascismes sociaux ", car c'est ainsi que j'appelle la conversion des inégalités économiques extrêmes en inégalités politiques, qui ne sont pas directement produites par le système politique de l'État capitaliste mais avec sa complicité. Par exemple, quand on privatise l'eau, l'entreprise propriétaire détient un pouvoir de veto sur la vie des personnes (s'ils ne paient pas leur facture, ils restent sans eau). C'est beaucoup plus qu'un pouvoir économique ou de marché. Bien que ce soit évident, cette crise ne facilite pas l'ouverture d'un espace pour l'émergence de nouveaux concepts de politique et de démocratie. Cette difficulté tient à deux choses : la prédominance des rapports capitalistes dont la reproduction exige aujourd'hui la coexistence entre la démocratie de basse intensité et les fascismes sociaux d'une part ; et, d'autre part, l'hégémonie de la démocratie libérale dans l'imaginaire social, souvent à 
travers le recours à des traditions ou mémoires historiques supposées qui la légitiment. Aucun de ces obstacles ne concerne Cuba. Il n'y pas plus de relations capitalistes qui dominent qu'une tradition libérale minimalement crédible. Il devrait ainsi être possible d'assumer la démocratie radicale comme point de départ, sans avoir à porter le poids de tout ce qui est dépassé dans l'expérience dominante de la démocratie de ces 50 dernières années.

2. De l'avant-garde à l'arrière-garde. Pour qu'une telle chose arrive, pour que la démocratie ne se réduise pas à un inventaire des acquis et aux argumentations rhétoriques mais arrive à se réaliser de façon systémique, un pas important doit être franchi : la conversion du parti d'avant-garde en un parti d'arrière-garde, qui soit moins un parti de direction qu'un parti de facilitation ; un parti qui promeuve la discussion de questions fortes, pour que dans la vie quotidienne des pratiques sociales les citoyens et les communautés soient mieux formés pour distinguer entre réponses fortes et réponses faibles. Un parti qui accepte naturellement l'existence d'autres formes d'organisation d'intérêts, avec lesquels il cherche à avoir une relation d'hégémonie et non une relation de contrôle. Cette transformation est la plus complexe de toutes et ne peut se réaliser que dans le cadre de l'expérimentation suivante.

3. Le constitutionnalisme transformateur. Les transitions dans lesquelles il y a des changements importants des rapports de pouvoir passent, en général, par des processus constituants. Lors des vingt dernières années plusieurs pays d'Afrique et d'Amérique latine ont vécu des processus de ce genre. Cette histoire récente nous permet de distinguer deux types de constitutionnalisme : le constitutionnalisme moderne proprement dit et le constitutionnalisme transformateur. Le constitutionnalisme moderne, qui a prévalu sans opposition jusqu'à récemment, est un constitutionnalisme construit par en haut, par les élites politiques, avec l'objectif de construire des États institutionnellement monolithiques et des sociétés civiles homogènes, ce qui a toujours impliqué la superposition d'une classe, une culture, une race, une ethnie, une région, sur les autres et au détriment des autres. Le constitutionnalisme transformateur part, au contraire, de l'initiative des classes populaires, comme une forme de lutte des classes, une lutte des exclus et leurs alliés, ayant pour objectif de créer de nouveaux critères d'inclusion sociale qui mettent fin à l'oppression de classe, raciale, ethnique, culturelle, etc. Une telle démocratisation sociale et politique implique la réinvention ou la refondation de l'État moderne. Une telle réinvention ou refondation ne peut éviter d'être expérimentale et cette caractéristique s'applique à la Constitution elle-même. Si possible, la nouvelle Constitution transformatrice doit avoir un horizon limité de validité, par exemple cinq ans, au terme desquels le processus constituant 
doit se rouvrir pour corriger les erreurs et intégrer des leçons de l'expérience. Limiter la période de validité de la nouvelle Constitution présente l'avantage politique - précieux en période de transition - de ne pas créer des gagnants ou des perdants définitifs. Cuba a des conditions idéales en ce moment pour rénover son expérimentation constitutionnelle.

4. État expérimental. De diverses manières, tant la crise terminale par laquelle passe le néolibéralisme que l'expérience récente des États les plus progressistes de l'Amérique latine, révèlent que nous nous acheminons vers une nouvelle centralité de l'État - une centralité plus ouverte à la diversité sociale (reconnaissance de l'interculturalité, de la pluriethnicité, et même de la plurinationalité, comme dans les cas de l'Équateur et de la Bolivie), économique (reconnaissance de différents types de propriété : étatique, communautaire ou commune (comunal), coopérative et individuelle) et politique (reconnaissance de différents types de démocratie, représentative ou libérale, participative, délibérative, référendaire, communautaire). Nous allons d'une centralité fondée sur l'homogénéité sociale vers une centralité fondée sur l'hétérogénéité sociale. Il s'agit d'une centralité régulée par le principe de la complexité. La nouvelle centralité opère de façon distincte dans des domaines où l'efficacité des solutions est démontrée (par exemple, à Cuba, l'éducation et la santé, malgré la dégradation de la qualité et de l'équité du système), dans des domaines où, au contraire, l'inefficacité est démontrée (à Cuba : accroissement des inégalités, agriculture) et dans les domaines nouveaux, qui sont les plus nombreux dans les processus de transition (par exemple, à Cuba : création de nouvelles institutions politiques, reconstruction de l'hégémonie socialiste sur la base d'une démocratie de haute intensité qui promeut simultanément la réduction de l'inégalité sociale et l'expansion de la diversité sociale, culturelle et politique). Pour les deux derniers domaines (domaines d'inefficacité démontrée et domaines nouveaux), il n'y a pas de recettes infaillibles ou de solutions définitives. Dans ces domaines, le principe de la centralité complexe suggère qu'on doit respecter le principe de l'expérimentation démocratiquement contrôlée. Le principe de l'expérimentation doit parcourir toute la société, et pour cela il faut que l'État devienne lui-même un État expérimental. Dans une phase de grandes mutations de la fonction de l'État et de la régulation sociale, il est inévitable que la matérialité de l'État, rigide comme elle l'est, sera sujette à de fortes vibrations qui en feront un champ fertile d'effets pervers, d'autant plus que cette matérialité institutionnelle est inscrite dans un espace-temps national/étatique qui souffrira de l'impact croisé des espaces-temps locaux et mondiaux.

Puisque la coexistence des solutions du vieux paradigme et du nouveau paradigme caractérise les époques de transition, et puisque les solutions du nouveau paradigme sont parfois aussi contradictoires entre elles que celles de l'ancien, je pense qu'il faut faire de l'expérimentation un principe de création institutionnelle, en particulier lorsque les solutions adoptées dans le passé se sont révélées inefficaces. Puisqu'il est imprudent d'adopter, lors de cette phase, des options institutionnelles irréversi- 
bles, l'État doit se transformer en champ d'expérimentation institutionnelle qui permet à différentes solutions institutionnelles de coexister et d'être en concurrence pour un temps, en tant qu'expériences pilotes, sujettes à un monitoring permanent par des collectifs de citoyens en vue de l'évaluation comparée des réalisations. La prestation de biens publics, surtout dans le domaine social, peut prendre diverses formes, et le choix entre elles, le cas échéant ${ }^{7}$, ne doit avoir lieu qu'une fois examinées par les citoyens leur efficacité et leurs qualités démocratiques.

Cette nouvelle forme d'un possible État démocratique de transition doit être basée sur trois principes d'expérimentation politique. Le premier est que l'expérimentation sociale, économique et politique exige la complémentarité entre diverses formes d'exercice du pouvoir démocratique (représentative, participative, communautaire, etc.). Aucune de ces formes prises séparément ne peut garantir que les nouvelles institutions soient efficacement évaluées. Il s'agit d'un principe difficile à respecter, surtout en raison de la présence complémentaire de divers types de pratique démocratique, elle-même novatrice et expérimentale. Dans ce contexte, il faut se rappeler la phrase d'Hegel : "Qui a peur de l'erreur a peur de la vérité ".

Le second principe est que l'État est authentiquement expérimental seulement dans la mesure où les différentes solutions institutionnelles bénéficient de conditions égales pour se développer selon leur propre logique. Autrement dit, l'État expérimental est démocratique dans la mesure où il donne une égalité des chances aux différentes propositions institutionnelles démocratiques. C'est uniquement ainsi que la lutte pour la démocratie devient véritablement une lutte pour des alternatives démocratiques. C'est uniquement ainsi qu'il est possible de lutter démocratiquement contre le dogmatisme d'une solution qui se présente comme la seule efficace ou démocratique. Cette expérimentation institutionnelle qui se produit dans le champ démocratique ne peut pas manquer de causer quelque instabilité et incohérence dans l'action étatique et de la fragmentation étatique qui peut en résulter éventuellement, peuvent surgir subrepticement de nouvelles exclusions.

Dans ces conditions, l'État expérimental ne doit pas seulement garantir l'égalité des chances aux différents projets de structure institutionnelle démocratique, mais doit également - et ce serait le troisième principe d'expérimentation politique - garantir des niveaux minima d'inclusion afin de rendre possible la citoyenneté active nécessaire pour contrôler et évaluer la réalisation des projets alternatifs. Conformément à la nouvelle centralité complexe, l'État combine la régulation directe des processus sociaux avec la méta-régulation, c'est-à-dire la régulation des formes étatiques de régulation sociale, dont l'autonomie doit être respectée, dès lors qu'elle respecte les principes d'inclusion et de participation consacrées par la Constitution.

5. Une autre production est possible. C'est un des domaines les plus importants de l'expérimentation sociale et Cuba peut jouer dans ce cadre un rôle de leadership stratégique dans la recherche de solutions alternatives, que ce soit aux modèles de développement capitaliste ou aux modèles de développement socialiste du XX ${ }^{\mathrm{e}}$ siècle. Au début du $\mathrm{XXI}^{\mathrm{e}}$ siècle,
7. Par exemple, des transports en commun d'État à côté de transports coopératifs ou de petites entreprises ; la production agricole en entreprises d'État à côté d'entreprises coopératives, communautaires ou de petits entrepreneurs capitalistes. 
la tâche qui consiste à penser et lutter pour des alternatives économiques et sociales devient particulièrement urgente pour deux raisons étroitement liées. En premier lieu, nous vivons une époque où l'idée qu'il n'y a pas d'alternatives au capitalisme a obtenu un degré d'acceptation probablement sans précédent dans l'histoire du capitalisme mondial. Deuxièmement, l'alternative systémique au capitalisme, représentée par les économies socialistes centralisées, s'est révélée inviable. L’autoritarisme politique et l'inviabilité économique des systèmes centralisés ont été mis en évidence de façon dramatique par l'effondrement de ces systèmes à la fin des années 1980 et au début des années 1990.

Paradoxalement, ces 30 dernières années le capitalisme s'est révélé comme jamais avant sa pulsion autodestructrice, de l'accroissement absurde de la concentration des richesses et de l'exclusion sociale à la crise environnementale, de la crise financière à la crise énergétique, de la guerre infinie pour le contrôle de l'accès aux ressources naturelles à la crise alimentaire. D'un autre côté, l'effondrement des systèmes du socialisme d'État a ouvert de l'espace politique pour l'émergence de multiples formes d'économie populaire, de l'économie solidaire aux coopératives populaires, des entreprises récupérées aux assentamentos issus de la réforme agraire, du commerce équitable aux modes d'intégration régional obéissant aux principes de réciprocité et de solidarité (comme l'ALBA). Les organisations économiques populaires sont extrêmement diverses et si certaines impliquent des ruptures radicales (quoique locales) avec le capitalisme, d'autres trouvent des formes de coexistence avec lui. La fragilité générale de toutes ces alternatives réside dans le fait qu'elles se donnent dans des sociétés capitalistes où les rapports capitalistes de production et de reproduction déterminent la logique générale du développement social, économique et politique. Pour cette raison, le potentiel émancipateur et socialiste des organisations économiques populaires finit par se bloquer. La situation privilégiée de Cuba dans le cadre de l'expérimentation économique réside dans sa capacité à définir, à partir de principes, logiques et objectifs non capitalistes, les règles du jeu selon lesquelles les organisations économiques capitalistes peuvent fonctionner. Pour réaliser tout le ferment de transition progressiste contenu dans le moment politique qu'elle vit, Cuba va avoir besoin de la solidarité de toutes les femmes et de tous les hommes, de toutes les organisations et mouvements de la gauche (au sens où nous l'entendons dans ce texte) du monde, et tout particulièrement du monde latino-américain. C'est le moment pour le monde de la gauche de rendre à Cuba tout ce qu'il lui doit pour être ce qu'elle est.

Texte traduit du portugais par Jim Cohen, relu par Nicolas Haeringer 